\title{
Affect Listeners. Acquisition of Affective States by means of Conversational Systems
}

\author{
Marcin Skowron \\ Austrian Research Institute for Artificial Intelligence, \\ Freyung 6, 1010 Vienna, Austria \\ marcin.skowron@ofai.at \\ http://www.ofai.at
}

\begin{abstract}
We present the concept and motivations for the development of Affect Listeners, conversational systems aiming to detect and adapt to affective states of users, and meaningfully respond to users' utterances both at the content- and affect-related level. In this paper, we describe the system architecture and the initial set of core components and mechanisms applied, and discuss the application and evaluation scenarios of Affect Listener systems.
\end{abstract}

\section{Introduction}

Emotional factors play an important role in intelligent behaviour; they influence perceptive, cognitive and communicative processes. In interactions between humans and artificial agents, the capability to detect signs of human emotions and suitably react to them can enrich interactions and improve their naturalness. Currently, interactive computer systems do not take into account the emotional dimension which humans expect to find in interaction, and this is a recurrent source of frustration [23]. Recently, there has been considerable interest in the development of artificial systems capable to detect and appropriately react to the users' behavior and emotional states. The recently started project SEMAINE [24] aims to develop Sensitive Artificial Listeners - conversational agents designed to sustain an interaction with a human user despite limited verbal skills, through robust recognition and generation of non-verbal behavior in real time. Other strands of research stress the importance of textual affect sensing, either motivated by the fact that current user interfaces in the human-computer interaction field are mainly text-based [9]; or by considering textual sentiment sensing as key for analyzing the users' sentiment towards specific products, news or movies as expressed e.g., in online postings [25].

The project CyberEmotions ${ }^{1}$ deals with modelling and understanding of the role of collective emotions in creating, forming and breaking-up of online-communities. In the field of social psychology, collective emotions have been defined as emotions that are shared by a large number of individuals in a certain society [29]. These emotions, experienced by

\footnotetext{
${ }^{1}$ http://www.cyberemotions.eu (all URLs last accessed 2009-11-15)
} 
individuals as reactions to societal and collective experiences, are shared between members of a group for a number of different reasons and are not limited to emotions which are felt by individuals as a result of their membership in a certain group (group-based emotions). Our research in the project focuses on the development of conversational systems that interact with members of various groups to probe for affective states and background knowledge related to those states for a large number of Internet users. We call this sort of systems Affect Listeners, which are not monolithic programs, but a family of systems with various constitutions, sharing the following characteristics: These systems communicate with users, rely on integrated affective components for detecting textual expressions of the users' affective states, and use the acquired information to aid selection and generation of responses. Affect Listeners monitor events and processes that draw attention of Internet users, by analysing a number of Internet websites (e.g., volksonomy-driven sites, automatic and semi-automatic news aggregators). These systems interact with users via a range of communication channels and interfaces (e.g., Internet Relay Chat (IRC), Jabber, online chat-site interface).

We aim at systems capable to adapt to the users' affective states, and to suitably respond to users' utterances both on the content- and the affect-related level. The foreseen evaluation scenario of the systems includes also their application in a laboratory for measuring physiological correlates of emotional responses occurring during interaction in experimental settings [7].

The rest of the paper is structured as follows: first, related work in the areas of affect computing, affect sensing from text, and dialog management is presented. Then, the Affect Listeners system architecture is introduced, along with a presentation of the components used in the initial realization of the system. Next a description of the core components related to affect detection and interaction management is provided. Finally the Affect Listeners testing and evaluation scenarios are presented.

\section{Related Work}

In recent years, we witnessed a growing interest in the development of human-agent interfaces that incorporate emotional behavior [18, 15]. Current research concentrates on the advances in embodied conversational agents [16] and speech-enabled animated characters. Research in this field included the creation of a framework to enrich human-agent interactions with an affective dimension and to verify under which conditions emotions can improve general intelligent behaviour of the synthetic characters, leading to more natural interactions between humans and computers [2]. The growth of academic research in affective computing resulted in the establishment of the EU-funded Network of Excellence "Human-Machine Interaction Network on Emotion" - HUMAINE ${ }^{2}$ aiming to contribute to "the development of systems that can register, model

\footnotetext{
${ }^{2}$ http://emotion-research.net
} 
and/or influence human emotional and emotion-related states and processes". Since 2004, the network and the subsequently established association have been providing a valuable basis for understanding the role of natural language processing with an emphasis on the modelling of affect in artificial systems.

A body of research relevant for work in Affect Listeners originates from works on affect sensing from text: a study on the extraction of affective components from texts and their application in dialog systems [30], analysis of blogposts [13], or assessing affect qualities of natural language text using large scale real-world knowledge [9]. The main strands of research on dialog management include finite state-based and frame-based approaches [3], plan based approaches [12], information state-based and probabilistic approaches [34]. Linguistic and psychological aspects have been studied in numerous works in the field of psycholinguistics. In [17] the authors, based on computer-aided text analysis, present evidence on links between word use and personality, social and situational fluctuations, and psychological interventions. [10] provides experimental results for the recognition of personality traits, on the basis of text conversations, utilizing both self and observer ratings of personality.

\section{System Architecture}

\subsection{Preconditions}

Application scenarios for Affect Listeners include online interactions with Internet users and the interaction with users in a laboratory environment as introduced above. The system-user communication is text-based, realtime and oriented at the detection and acquisition of users' affective states. The initial realization is an open-domain system, i.e., communication is not limited to a specific domain, topic or ICT-mediated community. The main requirements related to the foreseen application scenarios include:

- robust natural language-based communication,

- detection and classification of affective states based on text analysis,

- dialog and affective dialog management,

- modelling system-user interaction to facilitate the acquisition of affective states,

- system acceptance and usage by Internet users.

The system environment contains user utterances and textual data originating from websites. Considering the characteristics of the system environment and the requirements of the foreseen application scenarios, we adopted an interactive and incremental development approach to support redesign at later stages of development and flexibility in the addition of new tasks, components and mechanisms. Moreover, the testing and integration of existing components is prefered to reimplementation, in particular in the initial realizations of the system. 


\subsection{Initial Realization of the System}

The general system architecture includes 3 core layers: perception, control and actuator-communication. Here, we present an overview of the layers' functionalities and introduce the components and software tools which compose them; see sections 4 and 5 for a more detailed description of the core system components and mechanisms such as affect detection and classification related resources, Affect Listener Dialog Scripting commands (ALDS) or User Adaptation Mechanism.

The aim of the initial system realization was to create a sandbox for the integration and testing of components, and to develop the first version of the action selection and response generation components. Figure 1 presents the layers of the system architecture and the interaction loop with the environment.

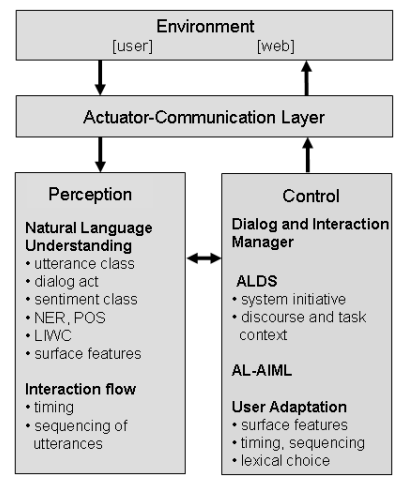

Fig. 1. Layers of the system architecture

Perception Layer: The perception layer integrates a number of natural language processing tools and machine learning-based classifiers for the analysis of both the input from the system's environment and the output of tools in the actuator layer, including the response candidates. The initial implementation includes the following components: maximum entropy based dialog act (DA) classifier ${ }^{3}$, maximum entropy and string similarity based utterance classifier [28] (UC) ${ }^{4}$, SVM-based question classifier $(\mathrm{QC})^{5}$, surface features detector (letter capitalization, punctuation,

\footnotetext{
${ }^{3}$ Dialog act classes follow annotation schema used in NPS Chat Corpus [5]: Accept, Bye, Clarify, Continuer, Emotion, Emphasis, Greet, No Answer, Other, Reject, Statement, System, Wh-Question, Yes Answer, Yes/No Question. The 10-fold cross validation accuracy is $71.2 \%$.

${ }^{4} 6$ utterance classes: greeting, question, rejection, agreement, goodbye, other.

${ }^{5}$ Classification scheme is based on the taxonomy proposed in [8], which consists of 6 coarse-grained and 50 fine-grained question classes. The classification accuracy of the component is $85.5 \%$ [27].
} 
emoticons), fine-grained answer candidate extraction [26] (NI) ${ }^{6}$, utterance focus and utterance interest detector[28], sentiment and affective states related classifiers and resources - Sentiment Classifier (SC) ${ }^{7}$, Linguistic Inquiry and Word Count (LC), (see section 4 for a more detailed overview of these affect detection and classification resources).

\author{
Utterance: hello George :) good to see you again. how are \\ you doing \\ - Annotation: UC-GREETING DA-whQuestion QC-DESCdef \\ LC-Social:CogMech:You:Time: SC-1 NS--1 PS-3 NI-PERSON-George
}

Tab. 1. Example annotation from the Perception Layer (excerpt).

\title{
Control Layer:
}

The control layer manages interaction with the user by analyzing information obtained from the perception layer e.g., timing of message exchanges, dialog act interpretation, utterance classes, sentiment valance and arousal. Furthermore, it monitors the dialog progression based on the system goals, observed dialog states, specific information contained in user utterance and system response candidates. The control layer is also responsible for selecting a system response from the number of response candidates (e.g., parallel response candidates from AL-AIML set), based on their annotation in the system's perception layer and a set of response selection rules. Finally, the control layer makes decisions on postprocessing of a final response and the timing of its dispatch, depending on the user profile and regularities discovered in his/her communicative style.

The Dialog and Interaction Manager is a central component in the Affect Listener system control layer. It is responsible for directing the system-user interaction to achieve the system goals. The component integrates the rule-based action selection, Affect Listener Dialog Scripting (ALDS), command interpreters for Artificial Intelligence Markup Language (AIML) and User Adaptation Mechanism (see section 5 for a detailed description of these components).

\section{Actuator-Communicator Layer:}

The actuator-communication layer includes a number of software tools, that provide information required for generating system responses, as well as for accepting/decoding a user input and for formating/dispatching system responses. The system communicates with users relying on a range of communication channels, mediated by the interface component. The initial realization of the Affect Listener system includes tools for accessing and processing collaborative tagging driven sites (Digg) and automatic news aggregators (Google News, Bing News), WordNet [4], User Adaptation Mechanism based system response postprocessing tool and communication interfaces (IRC, RPC, interface to online chat-websites).

\footnotetext{
${ }^{6}$ The system integrates Named Entity Recognition (NER) component with extraction rules and gazetteers for 50 distinctive answer type classes, that match the question classification taxonomy presented above.

7 The classifier provides the following annotations: sentiment class (SC), positive sentiment value (PS), negative sentiment value (NS).
} 


\section{Affect Detection and Classification}

The capability to detect and classify textual expressions of users affective states based on the analysis of their utterances is a core prerequisite for the Affect Listener systems. Recently, sentiment analysis of text and lexical affect sensing became a prominent and active research field [14] [19]. In the current realization of the Affect Listener system we use two affect detection and classification resources: Sentiment Classifier [31] and Linguistic Inquiry and Word Count (LIWC) dictionary [17]. In Affect Listener systems these resources are applied for processing both user utterances and the selected system responses (i.e., system responses generated based on text snippets retrieved from the Internet, for which no sentiment score can be calculated beforehand).

Sentiment Classifier. The system is implemented based on three classical classifiers: Naive Bayes, k-Nearest Neighbor and Centroid Document, and provides sentiment classes to text snippets. It is optimized for short textual messages (i.e., blog posts and comments) and detects and takes into consideration negation, exclamation marks, emoticons, intensifiers (i.e., "very" pleased), diminishers (i.e., "somewhat" agree) and text written in capital letters. The system provides positive/neutral/negative classification for a text snippet, and estimates its intensity on the scale between $[-5,5]$ and 0 for the neutral states. The average precision for the best result is $74 \%$ [31].

Linguistic Inquiry and Word Count (LIWC). The LIWC dictionary provides, among others, 32 word categories tapping psychological processes (e.g., social such as family, friends and human; affective such as positive and negative emotions; cognitive such as insight, causation, tentative), 22 linguistic processes (e.g., negations, adverbs, swear words), 3 paralinguistic dimensions (assents, fillers, nonfluencies), 7 personal concern categories (e.g., home, religion, work, leisure) for almost 4500 words and word stems [17]. The Affect Listeners' perception layer uses LIWC dictionary to annotate utterances according to those categories, thus allowing for generating representations of an individual utterance in terms of LIWC categories and, based on the aggregated input from a single agent (user or system), providing insights about its profile.

\section{Dialog and Interaction Management}

\subsection{Affect Listeners Dialog Scripting}

Affect Listeners Dialog Scripting (ALDS) commands facilitate the development of dialog scenarios, which require extended ${ }^{8}$ system-user message exchanges or fine-grained capabilities to detect cues in user utterances and system responses (natural language processing based analysis, affective states analysis) that cannot be captured by keyword or textual pattern-based matching mechanisms. The ALDS commands take advantage of perception capabilities of the system, including the textual affect sensing, classification of the dialog acts and extended parsing of the text.

\footnotetext{
${ }^{8}$ Measured in the number of exchanges and the scope of control over interaction.
} 
Dialog scenarios defined in ALDS are provided to the system in the form of text files, which describe the flow of a foreseen dialog scenario by specifying a set of conditional statements, perception channels and applicable actions required to generate system responses and to monitor the flow of the system-user message exchanges. A particular scenario is chosen based on the match between the current dialog state with the triggering conditions of a particular scenario. If found, the system enters the selected dialog scenario and attempts to progress the dialog according to a set of ALDS commands. Otherwise, the control layer manages the interaction based on other mechanism, i.e., rule based action selection or AL-AIML described below. The current version of ALDS includes:

- manipulation rules

- coarse grained processing commands: say, extract, verify if statement, use,

- fine grained processing commands: e.g., extract-dialogAct, extractentity, if-dialogAct, if-sentimentClass use-tool, use-store,

- symbols

- targets - (intended object of the processing): user utterance, system response, tool output, url,

- values - (object of the manipulation rules): sentimentClass-positive, dialogAct-agree, entity-locationCountry, tool-bingNews, etc.

ALDS scripts are e.g., used for realizing dialog scenarios focused on the acquisition of the user's attitudes and affective states in relation to the set of topics of interest of the user (detecting high positive (Tab. 2) or negative sentiment (Tab. 3) in a user's utterance, detecting topic of interest and affective states associate with it (Tab. 4)).

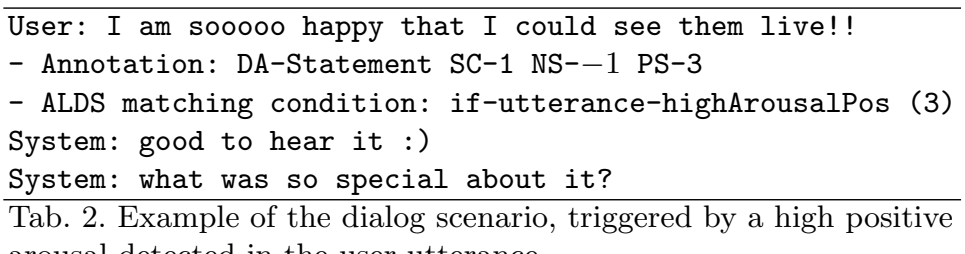
arousal detected in the user utterance.

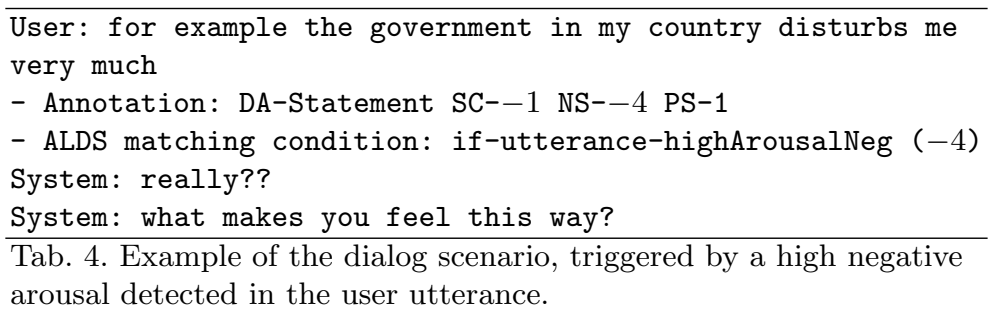

Tab. 4. Example of the dialog scenario, triggered by a high negative arousal detected in the user utterance.

ALDS scenarios are also used for gaining insights on the attitudes towards topics which are in the focus of attention of whole Internet communities (i.e., information on 'hot topics', which are automatically acquired through a set of tools from the system actuator layer from news aggregator websites such as http://news.google.com) and to strengthen 
communication cooperation with a user by providing the feeling of participation in joint activities [1] (e.g., initial message exchanges to introduce the system, if the user interacts with it for the first time).

The dialog scenario file consists of a number of command lines. Each command line describes a single user-system message exchange. The command line might further include an arbitrary number of sub-commands specifying the intermediate steps, actions applicable and if-then-else type statements, conditioning the realization of a consecutive sub-command based on true-false values returned for a particular statement. The final sub-command provides a system response generation instruction that incorporates symbols detected by the system in the ongoing dialog scenario. The ALDS commands are processed in a sequence until the exit condition is reached. The ALDS commands are extensible, i.e., new perception capabilities can be defined to add novel scenario triggers or conditioning parameters, without affecting the previously defined ones. Similarly, a new actuator tool can be used to specify and realize new dialog scenarios.

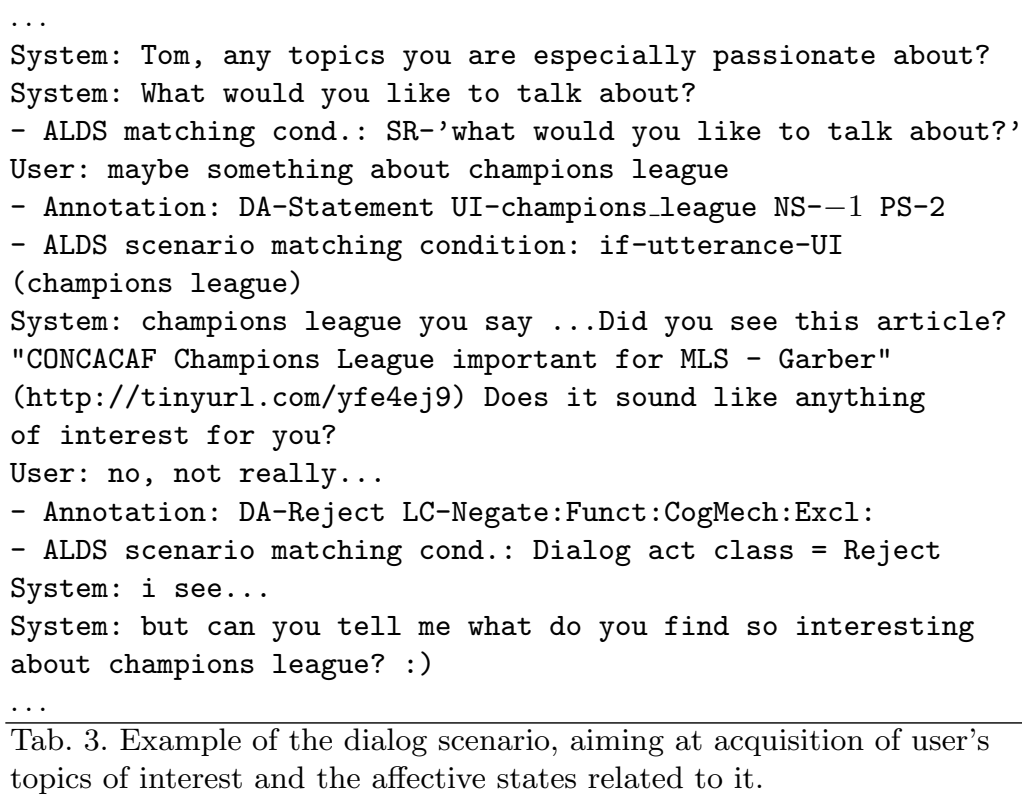

\subsection{Affect Listener AIML}

In the current realization of Affect Listener systems, the Affect Listener $\mathrm{AIML}^{9}$ set of response patterns (AL-AIML), provids a robust fall-back mechanism capable to generate system responses for a range of inputs which do not match activation cues of the ALDS scenarios or the rulebased action selection mechanism used by the Dialog and Interaction

\footnotetext{
${ }^{9}$ Artificial Intelligence Markup Language [32].
} 
Management. As presented above, the tasks of Affect Listener systems differ from tasks of domain specific dialog systems (e.g., ticket reservation, guidance systems). The requirements for Affect Listener applications include their capability to acquire users' affective states, while engaging in an open-domain, real-time communication with Internet users. While detection and acquisition of affective states is mediated by the system's perception layer, and mostly realized by specific affect state acquisition scenarios implemented in ALDS scripts, the capability to respond to a wide range of utterances is provided via an adapted AIML set. Response candidates generated based on AL-AIML are annotated in the perception layer and analysed by Dialog and Interaction Management component. System response is selected based on the dialog context and a set of response selection rules implemented in the control layer. The AL-AIML adaptation is based on the standard ALICE set [32], which was modified to provide character traits associated with active listening. The hypothesis is that such system characteristics will facilitate the acquisition of affective states. The AL-AIML set was created, aiming at encouragement of users to share information about their attitude, affective states and motivations, focusing on a user by mirroring questions and demonstrating the system's curiosity and interest; refraining from sounding too elaborate or providing extensive factoid knowledge about chatbot technology. This version of the AL-AIML set contains 14465 patterns, 15550 response instructions and 6918 srai substitution rules.

\subsection{User Adaptation Mechanism}

The User Adaptation Mechanism was introduced to facilitate establishing rapport with a user. Rapport can be defined as a feeling of connectedness that seems to arise from rapid and contingent positive feedback between partners and is often associated with socio-emotional processes. In [21], Reeves and Nass demonstrate that users prefer systems that become more like themselves over time over those which maintain a consistent level of similarity, even when the resultant similarity is the same. Gill et al. state that emphasizing commonalities and de-emphasizing differences is associated with increased solidarity and building rapport [6] and furthermore that this can also be achieved indirectly through the process of mirroring in which one person adopts some aspects of the behaviour of the other one. Our hypothesis is that establishing rapport with users via textual communication can be partially achieved by synchronizing with or mimicking the user's communication style as well as by providing textual back-channeling and signs of attention. This hypothesis, as well as the one, which postulates that adaptation to the user's communication style facilitates the acquisition of affective states will be tested in the experimental settings.

The described mechanism relies on the detection of user-specific textual communication features (perception layer), on monitoring a set of thresholds defined for deciding on the usage of selected synchronization and mimicking functionalities and the scope of their application (control layer), and on the postprocessing of system responses (actuator layer). The examples of mimicking and synchronization mechanisms include 
adaptation to a user regarding system response times, surface features of a user's communication style (e.g., usage of punctuation marks, capitalized or small letters), usage of emoticons or lexical choice. The decision on the application of a particular synchronization or mimicking functionality is made after the Dialog and Interaction Management component registers a consistency in a user's communicative style (e.g., a user uses emoticons more frequently than a predefined threshold). After a suitable postprocessing is chosen (e.g., usage of emoticons in the system responses based on the detection of strong valence in system responses [31]), the mechanism alters system responses as long as a particular aspect of a user's communication style remains constant.

\section{Evaluation Settings}

The evaluation scenario foreseen for Affect Listener systems include testing their performance in two tasks: realizing natural language-based communication and acquiring affective states from the users. System evaluation scenarios include tests with Internet users and tests with users in experimental laboratory settings capable to measure users' physiological emotional responses. The assessment of the system will be conducted by human assessors, and in an applicable scope, based on the automatic analysis of logs of system-user interactions.

Evaluation of the system by human assessors will provide data on users' subjective experiences from interactions with the system and the overall system performance in achieving its goals in the foreseen task (i.e., acquiring affective states of the users). For the Affect Listeners evaluation scenario we plan to perform the following types of human assessor-based tests:

- tests with users of a selected discussion group, followed by an online questionnaire where test participants will be asked to report their individual experience from interactions with the system in quantitative and qualitative ways,

- tests with Internet users, where user-system message exchanges will be accompanied by an input field through which users can select a numerical rating that represent their subjective feeling on the system's capability to preserve the dialog coherence, or users interest and satisfaction from the ongoing communication.

Manual evaluation of the system performance and the analysis of the system-user message exchange logs is a resource- and time-consuming process. On the other hand, automatic and complete evaluation of the conversational system performance extends beyond the state of the art. This does not, however, rule out the applicability of automatic and semiautomatic tests to assess selected system capabilities. The acquired data assists the ongoing iterative system development process, by supporting evaluation of the introduced mechanisms, and various realizations of the systems, including evaluation of a range of approaches for the natural language generation and dialog management.

Examples for measurements which can be automatically derived include the number of messages exchanged, the timing of the messages submitted 
by a user and the system, sentiment polarity associated with utterances. We are also investigating the scope of application of automatic methods for measuring dialog coherence, described in the literature [20]. Further, we perform automatic analysis of the system-user interaction logs based on the LIWC dictionary to (among others) gain insights on the quantity and types of expressions related to affective states that appear in the system-user interactions logs. In the context of the laboratory scenario presented in the introductory section, we plan to focus on measuring the emotional responses of users to the messages generated by the system and test the effectiveness of dialog scenarios and affect-related (i.e., detection, control and generation) mechanisms implemented in the system.

\section{Conclusions}

In this paper, we introduced the concept and the constitution of Affect Listeners. The presented set of implemented core components for the detection of textual expressions of users' affective states and for managing the progression of the dialog was integrated in the initial realization of the system. This version demonstrated the system's capabilities to detect and classify a user's textual expression of affective states and to direct a dialog in a way that facilates recognition of the user's topics of interest and the acquisition of background knowledge related to these topics.

The modularity and extensibility of the described system architecture allows for a flexible creation of different realizations of the Affect Listener systems. The development of these task-oriented conversational systems creates an opportunity for an extensive testing of various approaches for modelling system-user interactions in an open domain system that communicates with a wide range of users and via various communication platforms. This includes testing of the applicability of a range of dialog management and natural language generation components.

The system application scenarios include its usage for querying individual users about their affective states in relation to various entities, events and processes, including those automatically detected by the system based on the analysis of online resources and those discovered during the communication with a user. With the introduction of the real-time human-system interaction capabilities we focus on the detection of the current affective states of a large number of the individuals and create a platform, which allows for a selective acquisition of those states. The aggregated data shall provide a basis for extending our understanding of the affective states of individuals, groups of people, relations between the occurrence of external events and collective group feelings, and how those form and spontaneously evolve over time.

Acknowledgments. The work was supported by a European Union grant in the 7th Framework Programme, Theme 3: Science of complex systems for socially intelligent ICT. It is part of the CyberEmotions project (contract 231323). Additional supported has been provided by the Austrian Federal Ministry for Transport, Innovation and Technology and the Austrian Federal Ministry for Science and Research. 


\section{References}

1. Allwood, J. (1999); The Structure of Dialog. The Structure of Multimodal Dialogue II, Amsterdam, Benjamins, pp. 3-24.

2. Arafa, Y., et al. (2004); Affective Interactions for Real-time Applications: the SAFIRA Project. KI 18(1): 30, 2004.

3. Bohus, D., Rudnicky, A. (2003); RavenClaw: Dialog Management Using Hierarchical Task, Decomposition and an Expectation Agenda. In Proc. of the Eurospech 2003: 597-600.

4. Fellbaum, Ch. (1998); WordNet: An Electronic Lexical Database. Bradford Books.

5. Forsyth, E., Martell, C., (2007); Lexical and Discourse Analysis of Online Chat Dialog, Proc. of the First IEEE Int. Conf. on Semantic Computing, pp. 19-26.

6. Gill, D., Christensen and F. Fincham. (1999); Predicting marital satisfaction from behavior: Do all roads really lead to Rome? Personal Relationships 6: 369-387.

7. Kappas, A., Pecchinenda, A. (1999); Don't wait for the monsters to get you: A video game task to manipulate appraisals in real time. Cognition and Emotion, 13, 119-124.

8. Li, X. and Roth, D. (2002); Learning Question Classifiers, In Proc. of the 19th Int. Conf. on Computational Linguistics, pp. 556-562.

9. Liu, H., Lieberman, H., Selker, T. (2003); A model of textual affect sensing using real-world knowledge, In Proceedings Int. Conf. on Intelligent User Interfaces.

10. Mairesse, F., Walker, M., Mehl, M., Moore, R. (2007); Using linguistic cues for the automatic recognition of personality in conversation and text. Journal of Artificial Intelligence Research: 30, 457-500.

11. Mann, W., Thompson, S., (1988); Rhetorical structure theory: Rhetorical structure theory: Toward a functional theory of text organization. Text 8(3):243-281.

12. McGlashan S. (1996); Towards multimodal dialogue management, In Proceedings of Twente Workshop on Language Technology 11, The Netherlands.

13. Mihalcea, R., Liu, H. (2006); A corpus-based approach to finding happiness, in the AAAI Spring Symposium on Computational Approaches to Weblogs.

14. Neviarouskaya, A., Prendinger, Ishizuka, M. (2007); TextualAffect Sensing for Social and Expressive Online Communication, Affective Computing and Intelligent Interaction, Proc. Int'l Conf. ACII2007), pp. 218-229.

15. Paiva, A., Prada, R., and Picard, R.W. (2007); Affective Computing and Intelligent Interaction, Second Int. Conf., ACII 2007, Lisbon, Portugal.

16. Pelachaud C., Poggi I. (2001); Towards believable interactive embodied agents, Fifth Int. Conf. on Autonomous Agents workshop on Multimodal Communication and Context in Embodied Agents.

17. Pennebaker, James W., Mehl, Matthias R., Niederhoffer, Kate G. (2003); Psychological aspects of natural language use: our words, our selves., Annual Review of Psychology, Vol. 54: 547-577. 
18. Picard, R. (1997); Affective Computing, MIT Press, Cambridge, MA.

19. Prabowo, R., Thelwall, M. (2009); Sentiment Analysis: A Combined Approach, accepted for publication in Journal of Informetrics.

20. Purandare, A., Litman D. (2008); Analyzing Dialog Coherence using Transition Patterns in Lexical and Semantic Features.

21. Reeves, B. Nass, C. (1996); The Media Equation. Cambridge, Cambridge University Press.

22. Schank, R. C., and Abelson, R. (1977); Scripts, Plans, Goals and Understanding.

23. Schröder, M. Cowie, R. (2006); Developing a consistent view on emotion-oriented computing. In: Machine Learning for Multimodal Interaction, pp. 194-205.

24. Schröder, M., Cowie, R., Heylen, D., Pantic, M., Pelachaud, C., Schuller, B. (2008); Towards responsive Sensitive Artificial Listeners. Fourth International Workshop on Human-Computer Conversation.

25. Shaikh, M. A., Prendinger, H., and Ishizuka, M. (2008); Sentiment Assessment of Text by Analyzing Linguistic Features and Contextual Valence Assignment. Appl. Artificial Intelligence 22, 6, 2008.

26. Skowron, M. (2005); A Web Based Approach to Factoid and Commonsense Knowledge Retrieval, Doctoral Dissertation, Hokkaido University.

27. Skowron, M., Araki, K. (2005); Effectiveness of Combined Features for Machine Learning Based Question Classification, Special Issue on Question Answering and Text Summarization, Journal of Natural Language Processing, Vol. 6, pp. 63-83.

28. Skowron, M., Irran, J., Krenn, B. (2008); Computational Framework for and the Realization of Cognitive Agents Providing Intelligent Assistance Capabilities, (ECAI2008), The 6th Int. Cognitive Robotics Workshop, pp. 88-96.

29. Stephan, W. G., Stephan, C. W. (2000); An integrated threat theory of prejudice. In S. Oskamp (Ed.), Reducing prejudice and discrimination. Hillsdale, NJ: Erlbaum, 225-246.

30. Tatai, G., Laufer, L. (2004); Extraction of Affective Components from Texts and Their Use in Natural Language Dialogue Systems, Acta Cybernetica 16, 625-642.

31. Thelwall, M., Buckley, K., Cai, D., Paltoglou, G. (2009); CyberEmotions project: Internal Report - September 2009, University of Wolverhampton.

32. Wallace, R. (2001); Don't Read Me - A.L.I.C.E. and AIML Documentation, http://www.alicebot.com/dont.html.

33. Weischedel, R., Brunstein, A. (2005); BBN Pronoun Coreference and Entity Type Corpus, Linguistic Data Consortium, Philadelphia.

34. Williams J.D., Poupart P., Young S. (2005); Partially Observable Markov Decision Processes with Continuous Observations for Dialogue Management. In Proceedings of the 6th SigDial Workshop on Discourse and Dialogue.

35. Valitutti, A., Strapparava, C., Stock, O. (2004); Developing Affective Lexical Resources, PsychNology Journal, Vol. 2, No. 1. pp. 61-83. 